\title{
SMARTPHONE-BASED COLORIMETRIC DETERMINATION OF DPPH FREE RADICAL SCAVENGING ACTIVITY IN VEGETABLE OILS
}

\author{
*Sanita Vucane, Martins Sabovics, Lauris Leitans, Ingmars Cinkmanis \\ Latvia University of Life Sciences and Technologies, Latvia \\ *Corresponding author's email: sanitavucane@inbox.lv
}

\begin{abstract}
Free radicals can rapidly and irreversibly oxidize various structures, including unsaturated fatty acids in vegetable oils, which affect the sensory properties. Spectrophotometry is the most widely used method for the determination of free radical scavenging activity (RSA) using 2,2-diphenyl-1-picrylhydrazyl (DPPH). Barrier to the further use of classical analytical methods to analyze biologically active compounds in foodstuffs is that equipment requires high cost and has limited mobility. One of solutions is to replace classical methods, such as spectroscopy, with smartphonebased colorimetry. Huawei P30 Lite smartphone was used for colorimetric detection. The free radical scavenging activity (RSA) in vegetable oil was detected using an application 'Color Picker', with image matching algorithm for red, green, and blue (RGB) model. RSA was expressed as percentage and measured by the DPPH method. The aim of the study was to determinate the total free radical scavenging activity with smartphone-based colorimetry. For the data comparison and accuracy spectrophotometer as analytical optical instrument was used. Eleven vegetable oils: sea buckthorn, sunflower, rice, macadamia nut, hemp, corn, grape, linseed, rapeseed, olive and milk thistle oils were selected for analysis. The best results with no significant differences $(p>0.05)$ compared to smartphone-based colorimetry from spectrophotometry were determined using RG values. The poor results were detected by using B value $(p<0.05)$ and were not suitable for determination of RSA. Smartphone-based colorimetry can be used in the determination of the RSA in vegetable oils.
\end{abstract}

Key words: radical scavenging activity, digital image colorimetry, Android, Huawei, DPPH, vegetable oil.

\section{Introduction}

In analytical chemistry one of the most widely used methods for the determination of substances in coloured solution is spectroscopy. Spectroscopy has been a classical method for several years, but by the development of the smart digital equipment, there is a possibility to replace classical analytical methods with alternative methods. One of these alternatives is smartphones based on Android or iOS operating systems with good resolution cameras. Likewise, in spectroscopy, smartphone cameras and app algorithm can absorb the colour of light according to BeerLambert's law and can be used for colorimetric determination (Anderson, Bendell, \& Groundwater, 2004). Smartphone based analysis is used in chemical analysis (Coskun et al., 2012; Zhu et al., 2011, Masawat et al., 2015), paper-based strips (Yetisen et al., 2014), dermatology (Kroemer et al., 2011) and public health and safety (Jamalipour \& Hossain, 2019). The colour of visible light depends on its wavelength. Human eyes can recognize a distinctive colour in the visible light of each wavelength. Only three colours - red (R), green $(\mathrm{G})$ and blue $(\mathrm{B})$ are primary and are needed to make the white colour. Red, green, and blue colours are known as the RGB colour model to construct the visible light. RGB colour model digitally describes the intensity of the visible light in pixels ranging from 0 to 255 (Rhyne, 2016). The use of RGB model system with smartphone-based colorimetry would play an important role in the determination of antioxidants. Antioxidants inhibit oxidation of foodstuffs, increase the shelf life and retain the original taste and smell characteristics (Farhoosh, 2005; Rajeswer Rao,
2015). Arteriosclerosis and tumor formations are facilitated by free radical-caused cell membrane and deoxyribonucleic acid (DNA) damage (Phaniendra, Jestadi, \& Periyasamy, 2015). Free radicals can rapidly and irreversibly oxidize various structures, including unsaturated fatty acids that are components of the phospholipids in cell membranes (Jaswir, Che Man, \& Kitts, 2000; Reische, Lillard, \& Eitenmiller, 2002; Pelley, 2011; Rajeswer Rao, 2015). Plants, animal and human organisms have a strong defense system and physiological equilibrium between free radicals and antioxidants (Kelly et al., 1995). For the protection against radicals in living organisms, it requires enzymes (catalase, glutathione peroxidase), proteins, thiols, glutathione, and antioxidants that are mainly absorbed through food. During inflammation, physical fatigue causes an increase of the formation of free radicals that facilitate damage to this physiological equilibrium system (Miyashitau \& Takagi, 1986; Goffman \& Becker, 2001). It is proven that antioxidants are compounds that delay the formation of free radicals. In autooxidation processes oxygen in the air can spoil any fats that contain unsaturated fatty acids (Sies, 1991; Fürst, 1996; Abulude, Ogunkoya, \& Eluyode, 2005; Aparicio \& Harwood, 2013). Unsaturated fatty acids can easily react with air oxygen, and the product of primary oxidation is hydroperoxide that can actively react further. The speed of oxidation is greater, when there are more double bonds in fatty acid molecule (Wood et al., 2002). It is possible to delay the autooxidation by the treatment of fat, using suitable packaging or adding antioxidants that can help to retain the sensory properties (Chen, Shi, \& 
Ho, 1992). Antioxidants (AH), when they react with radicals R-and ROO', form a new and more stable radical $\mathrm{A}$, and the oxidation reaction is effectively slowed (Brand-Williams, Cuvelier, \& Berset, 1995; St.Angelo et al., 1996; Loftsson, 2014):

$$
\begin{gathered}
\mathrm{AH}+\mathrm{R} \cdot \rightarrow \mathrm{A} \cdot+\mathrm{RH} \\
\mathrm{AH}+\mathrm{ROO} \cdot \rightarrow \mathrm{ROOH}+\mathrm{A} . \\
\mathrm{A} \cdot+\mathrm{R} \cdot \rightarrow \mathrm{AR}
\end{gathered}
$$

The polyunsaturated fatty acids within the vegetable oil easily oxidize, giving the oil a bitter taste. It is important to have an ability to capture the free radicals in oils to stop degrading processes that can change the quality of oils and its sensory properties, which can reduce biologically active compounds and consequently nutritional value. Nowadays, analytical methods used to analyze biologically active compounds in foodstuff, require expensive equipment with limited mobility. Due to research and advances in nanoparticles and new information technologies, it has been made possible to produce simple, portable, and low-cost equipment. One of solutions to characterize food quality and determine various biologically active compounds is to replace classical optical methods, such as spectroscopy, with smartphonebased colorimetry. The smartphones can compete with classical optical methods due to their high-resolution cameras (image quality 48 megapixels, in future up to 108 megapixels), ultra-highly sensitive optical and light sensors, and determination according to BeerLambert law.

The aim of the study was to determine the total free radical scavenging activity with smartphonebased colorimetry.

\section{Materials and Methods}

The principle of the colorimetric analysis of the research object is the digital imaging of vegetable oils prepared for the determination of free radical scavenging activity with smartphone-based application for colour analysis with Red, Green, Blue (RGB) colour model.

Samples: In total, eleven vegetable oils: sea buckthorn, sunflower, rice, macadamia nut, hemp, corn, grape, linseed, rapeseed, olive, and milk thistle oils in the original commercial packaging were selected for analysis.

Equipment for analysis: Agilent Cary 60 UV/VIS Spectrophotometer (Agilent Technologies, Inc., US) was used for the comparison with the smartphone Huawei P30 Lite (Huawei Technologies Co., Ltd., China) measurement.

For imaging, the smartphone Huawei P30 Lite (Huawei Technologies Co., Ltd., China) released 2019, April 25, operating system EMUI 10 (Android 10), 48-megapixel triple camera were used.
Image acquisition system: The digital image acquisition system consisted of a polyvinyl chloride PULUZ photo studio (Puluz Technology Ltd., China) softbox $\left(24 \times 32 \times 38 \mathrm{~cm}^{3}\right)$. Constant light intensity was provided by a 40 pieces light-emitting diode (LED) lamp (model 2835), with luminous flux $550 \mathrm{~lm}$, colour temperature: $3200 \mathrm{~K}$ and power: $3.5 \mathrm{~W}$. LED lamp was located at the upper part inside the lightbox. For taking a colorimetric image, a smartphone with a 48-megapixel camera (Huawei P30 Lite) was positioned outside in front of open side of the box at a distance of $12 \mathrm{~cm}$ from the PS $2.5 \mathrm{~mL}$ macro disposable cuvettes (BrandTech Scientific, Inc., US) with sample, DPPH reagent or $96 \%$ ethanol solution.

Imaging and image analysis: Image was captured by the smartphone camera and saved as 8-bit JPG format with the average size of $7.0(8000 \times 6000$ pixels), ISO 400, f/1.8, $27 \mathrm{~mm}$ (wide), 1/2.0", 0.8 $\mu \mathrm{m}$, PDAF (Phase Detection Autofocus). The image was analyzed by a RGB colour model, application for Android 'Color Picker', which was installed from Android Apps on Google Play store. The image analysis system was used to relate the colour change of the sample with DPPH reagent.

Determination of total free radical scavenging activity using 2.2-diphenyl-1-picrylhydrazyl (DPPH): The DPPH total free radical scavenging activity in the vegetable oil was determined according to the method reported by Ahmed et.al. with slight modification (Ahmed, Khan, \& Saeed, 2015).

$D P P H$ reagent (Aldrich, Germany) with concentration $0.02 \mathrm{~g} \mathrm{~L}^{-1}$ was freshly prepared in $96 \%$ ethanol every day and kept at $4{ }^{\circ} \mathrm{C}$ in refrigerator, in a volumetric flask protected from light until further use.

Determination of free radical scavenging activity in vegetable oils: $0.1 \mathrm{~mL}$ of vegetable oil were mixed with $3 \mathrm{~mL}$ of DPPH reagent in $15 \mathrm{~mL} 120 \times$ $17 \mathrm{~mm}$ conical bottom PP tube (Sarstedt AG \& Co.KG, Germany) and vortexed $30 \mathrm{sec}$. with IKA Vortex 3 (KA $\circledR$-Werke GmbH \& Co. KG, Germany) by speed 7 . After 30 minutes of incubation in a dark place at room temperature $\left(21 \pm 1{ }^{\circ} \mathrm{C}\right)$, tubes were centrifuged (Pro-Research, Centurion Scientific Ltd., UK) at $3000 \mathrm{rpm}$ for 5 minutes and coloured solution was transfered to the PS $2.5 \mathrm{~mL}$ macro disposable cuvettes (BrandTech Scientific, Inc., US) with dimensions $12.5 \times 12.5 \times 45 \mathrm{~mm}$. Absorbance of the sample against blank $(96 \%$ ethanol) was measured at $517 \mathrm{~nm}$ using UV/VIS spectrophotometer Agilent Cary 60 (Agilent Technologies, Inc., US). Direct imaging of oil samples with DPPH reagent and $96 \%$ ethanol solution in cuvettes were captured using a smartphone-based colorimetric application by RGB colour model according to illustration of Figure 1. 


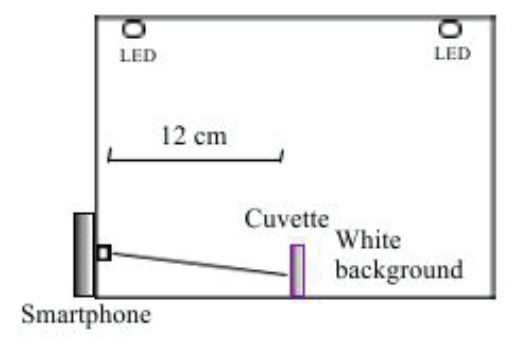

a.

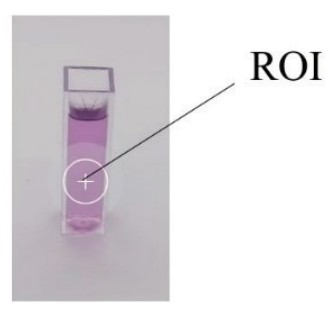

b.

Figure 1. (a) Illustration of photo studio lightbox experimental setup for image acquisition;

(b) example of region of interest (ROI) from a DPPH image.

Calculation method for determination of free radical scavenging activity with smartphone-based colorimetry

1) The obtained images from the smartphone application 'Color Picker' in RGB mode had an average colour value according to the equation reported by Jansons \& Meija (Jansons \& Meija,
2002) with modification for the individual red $\left(\mathrm{R}_{\mathrm{avg}}\right)$, green $\left(\mathrm{G}_{\text {avg }}\right)$, and blue $\left(\mathrm{B}_{\text {avg }}\right)$, red-green $\left(\mathrm{RG}_{\text {avg }}\right)$, redblue $\left(\mathrm{RB}_{\mathrm{avg}}\right)$, green-blue $\left(\mathrm{GB}_{\text {avg }}\right)$ and red-green-blue $\left(\mathrm{RGB}_{\mathrm{avg}}\right.$ ) colors by the equation 1, 2 and 3 .

2) The average colour value of $R_{\text {avg }}, G_{\text {avg }}, B_{\text {avg }}, R G_{\text {avg}}$, $\mathrm{RB}_{\mathrm{avg}}, \mathrm{GB}_{\mathrm{avg}}, \mathrm{RGB}_{\mathrm{avg}}$ was converted to absorbance by Beer-Lambert's equation 4 and 5 (Firdaus et al., 2014).

$$
\begin{aligned}
& R_{\text {avg }}=\frac{\sum_{i=1}^{n} R_{i}}{n} \\
& G_{\text {avg }}=\frac{\sum_{i=1}^{n} G_{i}}{n} \\
& \operatorname{red}\left(\mathrm{R}_{\mathrm{avg}}\right) \\
& \text { green }\left(\mathrm{G}_{\mathrm{avg}}\right) \\
& B_{\text {avg }}=\frac{\sum_{i=1}^{n} B_{i}}{n} \\
& R G_{\text {avg }}=\frac{\frac{\sum_{i=1}^{n} R_{i}}{n}+\frac{\sum_{i=1}^{n} G_{i}}{n}}{2} \\
& R B_{\text {avg }}=\frac{\frac{\sum_{i=1}^{n} R_{i}}{n}+\frac{\sum_{i=1}^{n} B_{i}}{n}}{2} \\
& \text { blue }\left(\mathrm{B}_{\mathrm{avg}}\right) \\
& \text { red-green }\left(\mathrm{RG}_{\mathrm{avg}}\right) \\
& \text { red-blue }\left(\mathrm{RB}_{\mathrm{avg}}\right) \\
& G B_{\text {avg }}=\frac{\frac{\sum_{i=1}^{n} G_{i}}{n}+\frac{\sum_{i=1}^{n} B_{i}}{n}}{2} \\
& \text { green-blue }\left(\mathrm{GB}_{\text {avg }}\right) \\
& R G B_{\text {avg }}=\frac{\frac{\sum_{i=1}^{n} R_{i}}{n}+\frac{\sum_{i=1}^{n} G_{i}}{n}+\frac{\sum_{i=1}^{n} B_{i}}{n}}{3} \\
& \text { red-green-blue }\left(\mathrm{RGB}_{\text {avg }}\right) \\
& A b s_{D P P H}=-\log \left(\frac{I_{D P P H}}{I_{0}}\right)
\end{aligned}
$$

where:

$I_{D P P H}-\mathrm{R}_{\text {avg }}, \mathrm{G}_{\text {avg }}, \mathrm{B}_{\text {avg }}, \mathrm{RG}_{\text {avg }}, \mathrm{RB}_{\text {avg }}, \mathrm{GB}_{\text {avg }}$ or $\mathrm{RGB}_{\text {avg }}$ average colour value of DPPH after 30 min $I_{0}-\mathrm{R}_{\text {avg }}, \mathrm{G}_{\text {avg }}, \mathrm{B}_{\text {avg }}, \mathrm{RG}_{\text {avg }}, \mathrm{RB}_{\text {avg }}, \mathrm{GB}_{\text {avg }}$ or $\mathrm{RGB}_{\text {avg }}$ average color value of the blank (96\% ethanol)

$$
A b s_{D P P H}=-\log \left(\frac{I_{o i l}}{I_{0}}\right)
$$

where:

$I_{\text {oil }}-\mathrm{R}_{\text {avg }}, \mathrm{G}_{\text {avg }}, \mathrm{B}_{\text {avg }}, \mathrm{RG}_{\text {avg }}, \mathrm{RB}_{\text {avg }}, \mathrm{GB}_{\text {avg }}$ or $\mathrm{RGB}_{\text {avg }}$ average colour value of vegetable oils with DPPH after 30 min $I_{0}-\mathrm{R}_{\text {avg }}, \mathrm{G}_{\text {avg }}^{\text {avg }}, \mathrm{B}_{\text {avg }}^{\text {avg }}, \mathrm{RG}_{\text {avg }}^{\text {avg }}, \mathrm{RB}_{\text {avg }}^{\text {avg }}, \mathrm{GB}_{\text {avg }}^{\text {avg }}$ or $\mathrm{RGB}_{\text {avg }}^{\text {avg }}$ avage colour value of the blank (96\% ethanol)

$$
R S A=\left(\frac{A b s_{D P P H}-A b s_{o i l}}{A b s_{D P P H}} * 100\right)=(\%)
$$

where:

$\mathrm{Abs}_{\mathrm{DPPH}}-\mathrm{R}_{\mathrm{avg}}, \mathrm{G}_{\mathrm{avg}}, \mathrm{B}_{\mathrm{avg}}, \mathrm{RG}_{\mathrm{avg}}, \mathrm{RB}_{\mathrm{avg}}, \mathrm{GB}_{\mathrm{avg}}$ or RGB avg absorbance of DPPH after $30 \mathrm{~min}$. $\mathrm{Abs}_{\text {oil }}-\mathrm{R}_{\text {avg }}, \mathrm{G}_{\text {avg }}, \mathrm{B}_{\text {avg }}, \mathrm{RG}_{\text {avg }}, \mathrm{RB}_{\text {avg }}, \mathrm{GB}_{\text {avg }}$ or $\mathrm{RGB}_{\text {avg }}$ absorbance of vegetable oil after $30 \mathrm{~min}$. 
3) Free radical scavenging activity (RSA) was calculated by equation 6 , according to the method reported by Ahmed et.al. with slight modification (Ahmed, Khan, \& Saeed, 2015).

Data Processing / Statistical Analysis

The data of the research was analyzed by the statistical and mathematical methods (mean, standard deviation). Data compared by the analysis of variance (ANOVA) and significance was defined at $p<0.05$. For the data analysis, the Microsoft Excel software version 2016 was used. Samples were analyzed in five repetitions.

\section{Results and Discussion}

The individual and mix colour values in RGB model system was explored to find out the best colorimetric detection of free radical scavenging activity (RSA, $\%$ ), because the CMOS image sensors in smartphones only detect red, green and blue colours (Kong et al., 2019). Natural white colour consists of all three colours R-red, G-green, and B-blue, therefore, colourwhite as calibration was used for background. Each primary color (Red, Green, Blue) in the RGB colour model has a pixel ranging from 0 to 255 and it means that the white colour has the same pixel range as RGB colours or 255. Theoretically, if white background is used, the average RGB should be 255 pixels, but research showed that for $96 \%$ ethanol solution using white background, the average RGB decreases to 180 pixels. Decrease in the pixel range is dependent on the concentration and used light, which is related to the used light in photography lightbox studio PULUZ with light-emitting diode (LED) colour temperature $3200 \mathrm{~K}$ and smartphone image parameters for the camera. Results in Table 1 show that sensitivity of blue (B) value differed from red (R), and green $(G)$ colours, except, Macadamia nut oil. Detected free radical scavenging activity of Macadamia nut oil by smartphone-based colorimetry was $47.3 \pm 0.6 \%$ and by UV/Vis spectrophotometry it was $45.9 \pm 0.1 \%$. The difference between used methods was only $1.4 \%$. Different sensitivity of B values was expressed with
$\mathrm{R}$ or $\mathrm{G}$, respectively RB and GB values, showed poor results $(\mathrm{p}<0.05)$ comparing to another $R G$ values and $\mathrm{UV} / \mathrm{Vis}$ spectrophotometry for all vegetable oils. Therefore, B, RB and GB values could not be used for the calculation of RSA for vegetable oils. 2,2-diphenyl-1-picrylhydrazyl (DPPH) is a stable free radical with scavenging capabilities of antioxidants from vegetable oils, which can donate a hydrogen to form the stable DPPH-H molecule. Absorbance decreased as colour changed from violet to pale yellow in the wavelength that ranged from 405 to 520 nm (Kumara, Sunil, \& Kumar, 2018). DPPH reacts with an antioxidant to form a yellow colour; however, maximum wavelength absorbance for classical UV/ Vis spectrometry is measured only by one spectrum at $517 \mathrm{~nm}$. Smartphone-based colorimetry can detect a wider visible light spectrum from 400 to $700 \mathrm{~nm}$ wavelength; therefore, complementary colour for analysis is needed (Turgeon, 2014). Colour wheel shows that complementary colour to violet colour is yellow (Figure 2), but only three colours red (R), green $(\mathrm{G})$ and blue (B) are primary and can be directly imaged with Android application 'Color Picker'; therefore, in case of the determination of RSA, a colour that closely matches the complementary yellow colour should be chosen. Green colour is close to primary colours, and to achieve better analytical results, a coloured-light mixing with primary colour red to obtain yellow colour should be used.

UV/VIS spectroscopy was used in comparison with smartphone-based colorimetry. Results show (Table 1) that analysis has a difference in range to $66.7 \%$ of RSA. The best results were obtained for RSA in RG values, and calculated $t$-value for smartphone-based colorimetry was lower than the critical $t$-value $(0.47$, $\mathrm{p}>0.05)$; therefore, no statistical difference at a $95 \%$ confidence level between the free radical scavenging activity by using the smartphone-based colorimetry and the UV/Vis spectroscopy for the analysis was observed. The highest free radical scavenging activity in vegetable oils was determined by UV/ Vis spectroscopy and Smartphone-based colorimetry

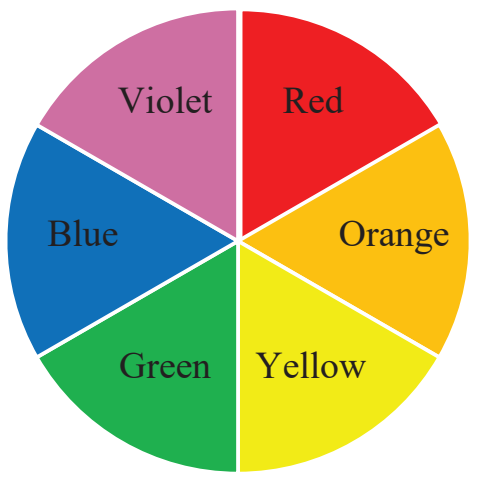

Figure 2. Colour wheel of primary and secondary colours. 
Comparison of free radical scavenging activity (\%RSA) by UV/Vis and Smartphone-based colorimetry with different color values from RGB modules

\begin{tabular}{|l|c|c|c|c|c|c|c|c|}
\hline \multirow{2}{*}{ Vegetable oils } & \multicolumn{7}{|c|}{ Method } \\
\cline { 2 - 9 } & UV-Vis & \multicolumn{7}{|c|}{ Smartphone-based colorimetry } \\
\cline { 2 - 9 } & & R & G & B & RG & RB & GB & RGB \\
\cline { 2 - 9 } Sea buckthorn & $93.5 \pm 0.1$ & $94.8 \pm 0.7$ & $91.7 \pm 0.7$ & $67.0 \pm 0.6$ & $93.2 \pm 0.7$ & $80.9 \pm 0.6$ & $79.3 \pm 0.6$ & $84.5 \pm 0.6$ \\
\hline Sunflower & $80.7 \pm 0.1$ & $85.2 \pm 0.6$ & $76.1 \pm 0.6$ & $40.6 \pm 0.6$ & $80.7 \pm 0.6$ & $62.9 \pm 0.6$ & $58.4 \pm 0.6$ & $67.3 \pm 0.6$ \\
\hline Rice & $67.6 \pm 0.1$ & $74.8 \pm 0.7$ & $59.5 \pm 0.7$ & $0.9 \pm 0.6$ & $67.2 \pm 0.6$ & $37.9 \pm 0.6$ & $30.2 \pm 0.6$ & $45.0 \pm 0.7$ \\
\hline $\begin{array}{l}\text { Macadamia } \\
\text { nut }\end{array}$ & $45.9 \pm 0.1$ & $66.8 \pm 0.6$ & $23.4 \pm 0.6$ & $47.3 \pm 0.6$ & $45.1 \pm 0.6$ & $57.0 \pm 0.6$ & $35.4 \pm 0.6$ & $45.8 \pm 0.6$ \\
\hline Hemp & $95.7 \pm 0.1$ & $96.0 \pm 0.7$ & $95.0 \pm 0.7$ & $68.1 \pm 0.6$ & $95.5 \pm 0.7$ & $82.0 \pm 0.6$ & $82.0 \pm 0.6$ & $86.4 \pm 0.6$ \\
\hline Corn & $61.5 \pm 0.1$ & $74.8 \pm 0.6$ & $47.9 \pm 0.8$ & $1.3 \pm 0.6$ & $61.3 \pm 0.6$ & $38.0 \pm 0.6$ & $24.6 \pm 0.6$ & $41.3 \pm 0.6$ \\
\hline Grape & $73.3 \pm 0.1$ & $84.2 \pm 0.6$ & $61.6 \pm 0.6$ & $34.4 \pm 0.6$ & $72.9 \pm 0.6$ & $59.3 \pm 0.6$ & $48.0 \pm 0.6$ & $43.4 \pm 0.6$ \\
\hline Linseed & $68.3 \pm 0.1$ & $77.4 \pm 0.6$ & $59.5 \pm 0.6$ & $15.3 \pm 0.6$ & $68.5 \pm 0.6$ & $46.4 \pm 0.6$ & $37.4 \pm 0.6$ & $52.3 \pm 0.6$ \\
\hline Rapeseed & $91.4 \pm 0.1$ & $89.7 \pm 0.6$ & $93.6 \pm 0.7$ & $46.1 \pm 0.6$ & $91.7 \pm 0.7$ & $67.9 \pm 0.6$ & $69.9 \pm 0.6$ & $76.5 \pm 0.6$ \\
\hline Olive & $95.7 \pm 0.1$ & $96.0 \pm 0.7$ & $95.0 \pm 0.7$ & $68.1 \pm 0.6$ & $95.5 \pm 0.7$ & $82.0 \pm 0.6$ & $82.0 \pm 0.6$ & $86.4 \pm 0.6$ \\
\hline Milk thistle & $94.3 \pm 0.1$ & $96.9 \pm 0.6$ & $92.2 \pm 0.6$ & $80.0 \pm 0.7$ & $94.6 \pm 0.6$ & $88.5 \pm 0.6$ & $86.1 \pm 0.6$ & $89.7 \pm 0.6$ \\
\hline
\end{tabular}

using a RG values, respectively, hemp 95.7 $\pm 0.1 \%$ $95.5 \pm 0.7 \%$, olive $95.7 \pm 0.1 \%-95.5 \pm 0.7 \%$, milk thistle $94.3 \pm 0.1 \%-94.6 \pm 0.6 \%$, sea buckthorn $93.5 \pm 0.1 \%$ $93.2 \pm 0.7 \%$ and rapeseed $91.4 \pm 0.1 \%-91.7 \pm 0.7 \%$ oils, but the lowest activity was detected in macadamia nut $45.9 \pm 0.1 \%-45.1 \pm 0.6 \%$, corn $61.5 \pm 0.1 \%-61.3 \pm 0.6 \%$ and rice $67.6 \pm 0.1 \%-67.2 \pm 0.6 \%$ oils. Precision of imaging method was evaluated by relative standard deviation (\%RSD). Using UV/Vis spectroscopy for the analysis, the value of RSD was lower and showed better sensitivity: $0.1 \%$, although the RSD for smartphone-based colorimetry was higher and ranged from 0.6 to $0.7 \%$. Unfortunately, in literature, there is lack of scientific researches reporting the determination of free radical scavenging activity using a smartphone-based colorimetry.

\section{Conclusions}

In the reported research, a new methodology for the determination of free radical scavenging activity based on the smartphone-based colorimetry was obtained. The principle of this method is to analyze a digital imaging of RSA in vegetable oils with DPPH, which is obtained with a smartphone camera, and show the results in application for colour analysis with RGB colour model using only RG values. Although UV/Vis spectroscopy has better sensitivity, the results showed that smartphone-based colorimetry in RG values can be used for the determination of RSA. Smartphone-based colorimetry is simple, portable, and low cost; therefore, it is necessary to develop new detection methods for various chemical analysis based on the principle of analysis of smartphone-based digital images.

\section{References}

Abulude, F.O., Ogunkoya, M.O., \& Eluyode, O.S. (2005). Effect of storage on the physicochemical properties of palm oil. Pakistan Journal of Scientific and Industrial Research. Pakistan Council of Scientific and Industrial Research (PCSIR), Karachi, Pakistan: 48:2, 5.ref. 110-112.

Ahmed, D., Khan, M.M., \& Saeed, R. (2015). Comparative Analysis of Phenolics, Flavonoids, and Antioxidant and Antibacterial Potential of Methanolic, Hexanic and Aqueous Extracts from Adiantum caudatum Leaves. Antioxidants 2015, 4, 394-409. DOI: 10.3390/antiox4020394.

Farhoosh, R. (2005). Antioxidant activity and mechanism of action of butein in linoleic acid. Food Chemistry, Vol. 93, Issue 4, 633-639. DOI: 10.1016/j.foodchem.2004.10.041.

Anderson, R.J., Bendell, D.J., \& Groundwater, P.W. (2004). Organic Spectroscopic Analysis. Royal Society of Chemistry; 1 edition, 8. Cambridge: The Royal Society of Chemistry.

Brand-Williams, W., Cuvelier, M.E., \& Berset, C. (1995). Use of a free-radical method to evaluate antioxidant activity. Lebensmittel-Wissenschaft und-Technologie., 28 (1), 25-30. DOI: 10.1016/S0023-6438(95) 80008-5. 
Chen, Q., Shi, H., \& Ho, C.T. (1992). Effects of rosemary extracts and major constituents on lipid oxidation and soybean lipoxygenase activity. Journal of the American Oil Chemists 'Society, Vol. 69 (10), 999-1002. DOI: $10.1007 / \mathrm{BF} 02541065$.

Firdaus, M.L., Alwi, W., Trinoveldi, F., Rahayu, I., Rahmidar, L., \& Warsito, K. (2014). Determination of Chromium and Iron Using Digital Image-based Colorimetry. Procedia Environmental Sciences, 20, 298304. DOI: 10.1016/j.proenv.2014.03.037.

Fürst, P. (1996). The role of antioxidants in nutritional support. Proceedings of the Nutrition Society, 55, 945961. DOI: 10.1079/PNS19960091.

Goffman, F.D., \& Becker, H.C. (2001). Diallel analysis for tocopherol contents in seeds of rapeseed. Crop Science, 41, 1072-1079.

Jamalipour, A., \& Hossain, M.A. (2019). Smartphone Instrumentations for Public Health Safety. Cham: Springer Nature Switzerland.

Janson, E., \& Meija, J. (2002). Kḷūdas kvantiatīvajās noteikšanās (Errors of quantitative determinations). Rīga: Rasa ABC. (in Latvian).

Jaswir, I., Che Man, Y.B., \& Kitts, D.D. (2000). Optimization of physicochemical changes of palm olein with phytochemical antioxidants during deep-fat frying. Journal of the American Oil Chemists'Society, Vol. 77 (11), 1161-1168. DOI: 10.1007/s11746-000-0182-6.

Kelly, F.J., Mudway I., Krishna, M.T., \& Holgate, S.T. (1995). The free radical basis of air pollution: focus on ozone. Respiratory Medicine, 89, 647-656. DOI: 10.1016/0954-6111(95)90131-0.

Kong, T., You, J.B., Zhang, B., Nguyen, B., Tarlan, F., Jarvi, K., \& Sinton, D. (2019). Accessory-free quantitative smartphone imaging of colorimetric paper-based assays. Lab on a Chip. 19(11), 1991-1999. DOI: 10.1039/ C9LC00165D.

Kroemer, S., Fruhauf, J., Campbell, T.M., Massone, C., Schwantzer, G., \& Soyer, H.P. (2011). Mobile teledermatology for skin tumour screening: diagnostic accuracy of clinical and dermoscopic image teleevaluation using cellular phones, British Journal of Dermatology 164. 973-979. DOI: 10.1111/j.13652133.2011.10208.x.

Kumara, P., Sunil, K., \& Kumar, A. (2018). Determination of DPPH Free Radical Scavenging Activity by RP-HPLC, Rapid Sensitive Method for the Screening of Berry Fruit Juice Freeze Dried Extract. Natural Products Chemistry \& Research. Volume 6, Issue 5, 1-7. DOI: 10.4172/2329-6836.1000341.

Loftsson, T. (2014). Autoxidation (auto-oxidation) is a complex oxidation mechanism that proceeds through a free radical chain process. Drug Stability for Pharmaceutical Scientists. Oxford: Academic press.

Miyashitau, K., \& Takagi, T. (1986). Study on the oxidative rate and prooxidant activity of free fatty acids. Journal of the American Oil Chemists' Society, 63, 1380-1384.

Pelley, J.W. (2011). Fatty Acid and Triglyceride Metabolism. Elsevier's Integrated Review Biochemistry (Second Edition). Philadelphia: Elsevier Sounders.

Phaniendra, A., Jestadi, D.B., \& Periyasamy, L. (2015). Free Radicals: Properties, Sources, Targets, and Their Implication in Various Diseases. Indian Journal of Clinical Biochemistry. 30(1), 11-26.

Rajeswer Rao, V. (2015). Chapter 7 - Antioxidant Agents. Advances in Structure and Activity Relationship of Coumarin Derivatives. London: Academic Press.

Reische, D.W., Lillard, D.A., \& Eitenmiller, R.R. (2002). Antioxidants. In: Akoh, C.C. and Min, D.B., Eds., Food Lipids: Chemistry, Nutrition and Biotechnology. New York: Marcel Dekker.

Rhyne, T.M. (2016). Applying Color Theory to Digital Media and Visualization. Boca Raton: CRC Press

Sies, H. (1991). Oxidative stress II. Oxidants and Antioxidants. London: Academic Press.

St.Angelo, A.J., Vercellotti, D.J., Jacks, T., \& Legendre, M. (1996). Lipid oxidation in foods. Critical Reviews in Food Science and Nutrition 36, 175-224. DOI: 10.1080/10408399609527723.

Turgeon, M.L. (2014). Linne \& Ringsrud's Clinical Laboratory Science: The Basics and Routine Techniques. Missouri: Elsevier Mosby.

Wood, L.G., Fitzgerald, D.A., Lee, A.K., \& Garg, M.L. (2002). Improved antioxidant and fatty acid status of patients with cystic fibrosis after antioxidant supplementation is linked to improved lung function. American Journal of Clinical Nutrition, 77, 150-159. DOI: 10.1093/ajcn/77.1.150.

Yetisena, A.K., Martinez-Hurtadoa, J.L., Garcia-Melendrezb, A., da Cruz Vasconcellosa, F., \& Lowea, C.R. (2014). A smartphone algorithm with inter-phone repeatability for the analysis of colorimetric tests. Sensors and Actuators B 196, 156-160. DOI: 10.1016/j.snb.2014.01.077. 